\title{
Probabilistic Analysis of Creep Crack Initiation and Growth in Pipe Components
}

\author{
Nikbin, K. M., Yatomi, M., Wasmer, K. and Webster, G. A. \\ Department of Mechanical Engineering \\ Imperial College London \\ South Kensington Campus, \\ London, SW7 2AZ, UK
}

\begin{abstract}
In this paper creep data have been collected on uniaxial specimens of a carbon manganese (C-Mn) steel and a $9 \% \mathrm{Cr}$ steel, designated $\mathrm{P} 91$, at 360 and $625^{\circ} \mathrm{C}$, respectively. Additional tests have been performed at these temperatures on precracked compact tension (CT) specimens and pressurized pipes and tubes containing axial cracks to measure the creep crack initiation (CCI) and creep crack growth (CCG) rate properties of these steels. All the data have been analysed statistically assuming log-normal distributions in properties. The results of this analysis have been incorporated into a Monte-Carlo simulation to predict the cracking behaviour of the pressurized pipes from the uniaxial and 'benchmark' CT specimen data. Good agreement has been obtained for the P91 steel but not for the C-Mn steel. The discrepancy observed for the C-Mn steel has been attributed to constraint effects, possible extensive plasticity which occurred on loading and to uncertainties in the determination of the creep fracture mechanics parameter $C^{*}$ used for characterising the cracking behaviour. Further comparisons have been made with deterministic calculations involving different combinations of material properties in a sensitivity study to establish probabilities of failure in the pipes based on the 'benchmark' CT specimen.
\end{abstract}

Keywords: creep, crack growth rate, crack initiation, fracture mechanics, $K, C^{*}$, limit analysis, reference stress, high temperature testing, Monte Carlo, steels, constraint.

\section{Introduction}

For design and safety assessment purposes it is often necessary to establish the significance of defects in components that are subjected to creep and creep/fatigue loading. Several procedures are available [1-5] for this purpose. When these procedures are used at the design stage, the sizes of defects postulated are determined by the resolution of non-destructive inspection methods. Otherwise the sizes of defects detected in service are used to make estimates of remaining lifetimes. It is essential that the procedures are reliable and sufficiently conservative.

The procedures assume that creep crack initiation (CCI) and creep crack growth (CCG) rate are correlated by the creep fracture mechanics parameter $C^{*}$. Normally for components this term is estimated by reference stress methods [6-7]. However, when CCI 
and CCG properties are being determined in the laboratory from compact tension (CT) specimens, experimental methods are employed to calculate $C^{*}[8]$.

In this investigation the significance of scatter in the measured creep properties is examined using statistical analysis. Data are reported on uniaxial creep specimens, CT test-pieces and pressurized pipes and tubes which contain external axial cracks [9, 10]. Deterministic calculations are performed, using combinations of upper bound, lower bound and mean material properties values taken from the uniaxial and 'benchmark' CT data, to predict the CCI and CCG behaviour of the pipes and tubes and make comparisons with Monte-Carlo simulations to obtain probabilities of failure.

\section{Fracture mechanics at high temperatures}

In general, plastic deformation and creep can be described by the same type of power law expression. For plasticity, plastic strain $\varepsilon$ can be expressed in terms of stress $\sigma$ by

$$
\varepsilon=A \sigma^{N}
$$

and creep strain rate $\dot{\varepsilon}$ by

$$
\dot{\varepsilon}=C \sigma^{n}
$$

where $A, C, N$ and $n$ are material constants. Normally equation (2) is used to describe secondary creep. When primary, secondary and tertiary creep are all observed it can be modified to describe an average creep rate $\dot{\varepsilon}_{A}$ so that

$$
\dot{\varepsilon}_{A}=\frac{\varepsilon_{f}}{t_{r}}=C_{A} \sigma^{n_{A}}
$$

where $\varepsilon_{f}$ is the uniaxial creep failure strain, $t_{r}$ is the time to rupture and $C_{A}$ and $n_{A}$ are material constants.

For a cracked body the stress field generated at a crack tip is characterised by $J$ when plastic strains dominate [11]. When creep dominates it is characterised by $C^{*}[8,12-16]$. For the case when $N=n$, identical stress fields are produced for the plastic and creep situations and similar methods can be employed to calculate both $J$ and $C^{*}[13]$.

For CT specimens ASTM E1457-01 [8] allows $C^{*}$ to be calculated from creep load line displacement rate $\dot{\Delta}^{c}$ using the expression

$$
C^{*}=\frac{P \dot{\Delta}^{c}}{B_{n} b} F
$$


where $P$ is the applied load, $b$ is the remaining ligament ahead of the crack and $B_{n}$ is the net thickness between side-grooves when used. Here, $F$ is a factor which depends on geometry and creep stress index, $n$, and is given by

$$
F=\frac{n}{n+1}[2+0.522(1-a / W)]
$$

It is important to note that the accepted procedure in the codes [1-2,4-5] is to estimate residual lives in components from 'benchmark' data derived from CT specimens using the $C^{*}$ approach [12-14].

In components where a formula similar to equation (4) is not available, $C^{*}$ must be determined by finite element methods or reference stress concepts [6-7, 13]. When the reference stress approach is adopted

$$
C^{*}=\sigma_{r e f} \cdot \dot{\varepsilon}_{r e f}\left(\frac{K}{\sigma_{r e f}}\right)^{2}
$$

where $K$ is the stress intensity factor and $\dot{\varepsilon}_{\text {ref }}$ is the uniaxial creep strain rate at the appropriate reference stress, $\sigma_{r e f}$. Ideally, $\dot{\varepsilon}_{r e f}$ should be taken from uniaxial data obtained on the same batch of material.

Usually it is convenient to employ limit analysis [17],[18] to obtain $\sigma_{r e f}$ from

$$
\sigma_{r e f}=\sigma_{y} \frac{P}{P_{l c}}
$$

where $P_{l c}$ is the collapse load of the cracked body and $\sigma_{y}$ is the yield stress. The value of $P_{l c}$, and hence estimates of $C^{*}$ in equation (6), will depend on the collapse mechanism adopted and whether plane stress or plane strain conditions are assumed. Both 'Global' and 'Local' collapse mechanisms can be chosen [19]. Collapse load solutions are available for many geometries [e.g. 1-4,19-20], but in some cases need to be derived numerically.

Previously it has been shown [21] that a 'Global' solution of reference stress [4] is most appropriate for cracked pipe components and therefore this solution is chosen in this paper to estimate $C^{*}$. 'Global' solutions are based on collapse of the entire cross-section at the site of the defect. For a semi-elliptical axial defect in a pipe subjected to an internal pressure $p, \sigma_{r e f}$ can be expressed as [4]

$$
\sigma_{\text {ref Global }}=\frac{p}{\frac{1}{R_{e}-a} \cdot \operatorname{bate}(a, c)+\ln \left(\frac{R_{e}-a}{R_{i}}\right)}
$$


where $\operatorname{bate}(a, c)$ is given by

$$
\text { bate }(a, c)=\frac{a}{\sqrt{\left.1+1.61 \cdot \frac{c^{2}}{\left[\left(R_{e}-a\right) \cdot a\right.}\right]}}
$$

In these equations, $a$ is crack depth, $c$ is half crack length at the surface and $R_{i}$ and $R_{e}$ are the internal and external radii of the pipe, respectively.

\section{Characterisation of creep crack initiation and growth}

Arguments for correlating high temperature crack initiation and growth essentially follow those of elastic-plastic fracture mechanics methods. For situations where creep dominates, it has been found that $[8,12-16]$ crack growth rate $\dot{a}$ can be described by

$$
\dot{a}=D C^{* \phi}
$$

where $D$ and $\phi$ are material constants that can be measured experimentally or determined from uniaxial creep data [4,12-16,22].

Prior to the onset of steady state creep crack growth that is described by equation (10), usually an incubation period is observed during which damage builds up at the crack tip [23]. The duration of this period is determined to some extent by the resolution of the crack monitoring equipment used to detect it. Typically this corresponds to a crack extension $\Delta a$ of between 0.1 and $0.5 \mathrm{~mm}$ depending on component shape and size. In this study an extension of $0.5 \mathrm{~mm}$ is adopted as relevant to defects in pipes. From equation (10) it may be expected that the time $t_{i}$ to initiate an extension of $\Delta a$ is given by an expression of the form.

$$
t_{i}=D_{i} \cdot C *-\phi_{i}
$$

where $\phi_{i}$ and $D_{i}$ are material constants. A lower bound to $t_{i}$ can be obtained by assuming that steady state cracking dominates in this period so that $\phi=\phi_{i}, D_{i}=\Delta a / D[24]$ and the incubation time becomes

$$
t_{i}=\frac{\Delta a}{D} \cdot C^{*-\phi}
$$

Equations (10)-(12) will be used in conjunction with equations (8) and (9) to make predictions of CCI and CCG in the pressurized pipes and tubes. 


\section{Probabilistic Analysis}

Uniaxial creep properties and CCI and CCG rate data are subject to experimental scatter. In order to quantify risk of failure, statistical analysis of the data is required [25]. Alternatively, a sensitivity study can be carried out using deterministic calculations with different combinations of upper, mean and lower bound material properties. When a statistical analysis is undertaken equations (2) or (3), (10) and (11) are re-written as;

$$
\begin{aligned}
& \dot{\varepsilon}=10^{\log \left(C^{\prime}\right)} \sigma^{n+e_{n}} \cdot 10^{e_{i}} \\
& \dot{a}=10^{\log \left(D^{\prime}\right)} \cdot C^{*^{\phi+e_{\phi}}} \cdot 10^{e_{i}} \\
& t_{i}=10^{\log \left(D_{i}^{\prime}\right)} \cdot C^{*^{\phi_{i}+\phi_{\phi_{i}}}} \cdot 10^{e_{t_{i}}}
\end{aligned}
$$

where $e$ with the appropriate subscript is the error in the variables which are obtained from the experimental data and statistical analysis. In equations (13)-(15), $e_{n}, e_{\phi}$ and $e_{\phi_{i}}$ are normal standard distributions derived form the scatter in $n, \phi$ and $\phi_{i}$ and $e_{\dot{\varepsilon}}, e_{\dot{c}}$ and $e_{t_{i}}$ corresponds to the normal error distribution in creep strain rate, creep crack growth rate and creep initiation time respectively. Finally $C^{\prime}, D^{\prime}$ and $D_{i}^{\prime}$ in equations (13)-(15) are the value of $C, D$ and $D_{i}$, respectively, in equations (2), (10) and (11), which ensure that the mean line fitting the experimental results always pass through the centroid of the available data set (as shown for example in Fig. 1) when slopes of $n+e_{n}, \phi+e_{\phi}$ and $\phi_{i}+e_{\phi_{i}}$ are adopted.

It should be noted from Fig. 1 that when the data are generated from CT specimens, $C^{*}$ is determined experimentally and the statistical analysis is assumed to be accurate. While, for components when $C^{*}$ is calculated from equation (6), it will include the variability in strain rate given by equation (13) so that estimates of $\dot{a}$ will not only depend on scatter in the data from Fig. 1 but also variability in $C^{*}$ as illustrated in Fig. 2. This combination of variabilities is discussed later in the analyses using a Monte-Carlo simulation. In this simulation calculations are carried out for combinations of values of the variables (chosen randomly from their statistical distributions) to produce probabilities of times to crack initiation or a specific amount of crack extension in a pressurized cracked pipe. In each case, 10,000 calculations were made to obtain probabilities of failure in the 'tails' of the cumulative distribution function.

\section{Experimental Data}

Experimental data from three European collaborative projects [9-10,26-27] have been examined. The materials tested were a C-Mn steel and a $9 \% \mathrm{Cr}$ steel (P91) with the compositions given in Table 1 . They were tested at respectively 360 and $625^{\circ} \mathrm{C}$. Their tensile properties at these temperatures are given in Fig. 3 and Table 2. Their corresponding creep strain rate data are shown in Figs. 4 and 5 and Table 3 which also lists the uncertainties in $C, n$ and $\varepsilon_{f}$ obtained from a statistical analysis of the results. The stresses applied during creep for the C-Mn steel were above the yield stress and significant plasticity occurred on loading. There was also appreciable primary and tertiary 
creep and average creep strain rate has been recorded for this material in Fig. 4 and Table 3. For the P91, stresses were below yield and mainly secondary creep was observed. It is evident from the results that much less variability was obtained from the creep properties of P91 and this is reflected in the smaller standard deviations calculated in the table.

Creep crack initiation and CCG rate data obtained from the collaborative projects [2627] for each material are shown in Figs. 6 to 9. The corresponding mean and standard deviations obtained from statistical analysis of the results are listed in Tables 4 and 5 . The data were collected from tests on CT specimens that were performed according to ASTM E1457-01 [8] standard specifications. It is apparent from the tables that similar standard deviations are calculated for both materials. For the C-Mn steel three sizes of specimen were examined and no distinction in the results was found. There is more scatter in the initiation data (Fig. 7) than in crack growth rate (Fig. 6) for this material. This is attributed to the difficulty in accurately determining crack initiation. For the P91 material (Figs. 8 and 9) some slow cyclic (frequency $f<0.01 \mathrm{~Hz}$ ) data have been included. Little effect on cracking rate (Fig. 8) is observed but it is apparent that cycling shortens the initiation time (Fig. 9). Also included in Figs. 7 and 9 are predictions of CCI using CCG rate data and equation (12). Approximate agreement with the means time in Fig. 7 for the $\mathrm{C}-\mathrm{Mn}$ steel is obtained suggesting that cracking during the incubation period is close to the steady state CCG rate. For the P91 steel, equation (12) approximately corresponds with the -2SD prediction suggesting that damage takes some while to build up at a crack tip in this material.

Results of CCI and CCG tests on the pressurized tubes and pipes have been reported previously [26, 27-29]. The dimensions of the pipes and tubes and pressures applied are given in Table 6. Also included are the dimensions. All the defects were axial and introduced on the outside. For P91 the defects were approximately semi elliptical; for the C-Mn steel they were nearly rectangular and represented long shallow slits. The results are shown in Figs. 10-13. In calculating $C^{*}$ for the pipes and tubes in these figures, $C^{*}$ was determined from equation (6) using equations (8) and (9) and the mean creep strain rate properties taken from Figs. 4 and 5. In the P91 steel, cracking proceeded along the surface (c) and radial (a) directions so that the crack maintained approximately the same semi-elliptical shape. For the C-Mn steel cracking only grew in the radial (a) direction. From Figs. 10 and 11 it is clear for the C-Mn material that cracking initiates sooner in the tubes and pipes and progresses faster than it does for $\mathrm{CT}$ specimens. There are a number of possible causes. The large difference can be due to constraint effects, the material properties used and the method of $C^{*}$ estimation. For example no allowance has been made for plasticity when determining $C^{*}$ from displacement rates in the experimental relation equation (4) when plotting the CT data. This could result in these $C^{*}$ values being over-estimated. In addition, the formulae used (equations (8) and (9)) for calculating $C^{*}$ in the tube and pipe are for semi-elliptical cracks, whereas the defects more closely resembled rectangular slits. Nevertheless use of a references stress $\sigma_{r e f}$ based on 2 dimensional axial cracks although giving improved correlation, still does not give close agreement. Also with $n=12.5$ for this material, only a small error in $\sigma_{r e f}$ could translate into a large error in $C^{*}$. The situation is different for the P91 steel. In this material close correspondence is achieved between the CT and pipe results. The standard deviations for CCG and CCI for the tubes and pipes for both materials are given in Tables 
7 and 8 , respectively. It is apparent that they are very close to those for the CT specimens as is to be expected, assuming that the same processes control cracking in each case.

Since it has been shown from analysis of the experimental results in Tables 4 and 5, and 7 and 8 that the same values of $\phi$ and $\phi_{i}$ can be chosen for the CT specimens and cracked pipes and tubes for both materials (note that for C-Mn steel $\phi$ can be chosen at lower value of $C^{*}$, which gives conservative estimation of CCG rate at higher value of $\left.C^{*}\right)$, a scaling factor approach is proposed for predicting the behaviour of the pipes and tubes from the 'benchmark' CT data. This can be achieved by estimating the cracking rate in the cracked pipe $\dot{a}_{c p}$ from equation (10) using

$$
\dot{a}_{c p}=\kappa_{s} D C^{* \phi} .
$$

where $\kappa_{s}$ is a scaling factor which superimposes the cracked pipe and 'benchmark' CT CCG rate data shown in Figs. 10 and 12. Similarly for the initiation time of the cracked pipe, $t_{i c p}$, equation (11) can be re-written as

$$
t_{i c p}=\kappa_{i} D_{i} \cdot C^{*-\phi_{i}} .
$$

where $\kappa_{i}$ is the scaling factor which superimposes the cracked pipe and 'benchmark' CT CCI data shown in Figs. 11 and 13. For both CCG and CCI, the scaling factors are used to allow for differences in constraint between specimen types and different methods of calculating $C^{*}$. A scaling factor close to one implies close agreement between the cracked pipe and $\mathrm{CT}$ experimental results.

The actual values obtained for $\kappa_{s}$ and $\kappa_{i}$ for each material are shown in Tables 7 and 8 . It is evident that agreement within a factor of about 2 between the cracked pipe data and CT results is obtained for P91 steel. For the C-Mn steel, the 'benchmark' CT specimen CCG rate has to be elevated by about 15 times and the CCI time reduced by approximately $1 / 4$ to achieve correspondence with the cracked pipe and tube results. It is also evident from the P91 data that although the CCG static and cyclic data have the same scaling factor, different values are needed for the CCI data. Appropriate use of the scaling factors based on Tables 7 and 8 will ensure accurate predictions of cracked pipes and tube behaviour from 'benchmark' CT data.

\section{Sensitivity Analysis and Monte-Carlo Simulation}

When making predictions of component lifetimes by deterministic methods it is common practice to undertake a sensitivity analysis to establish the importance of changes in material properties, loading conditions, component dimensions and crack size. In this investigation choice of material properties and whether an incubation period is included or not in the calculations, are considered for examining the time taken for a crack to grow a specified amount in the C-Mn steel and P91 pipes. In making the calculations, the uniaxial creep properties given in Table 3 have been employed to determine $C^{*}$ and the CT specimen data provided in Tables 4 and 5 to estimate CCG and CCI using the scaling 
factors listed in Tables 7 and 8. In the deterministic calculations, combinations of materials properties listed in Table 9, where $\pm 2 \mathrm{SD}$ values have been chosen to represent bounding behaviour, have been selected and applied to the pipe geometries and loading conditions given in Table 10. The results are included in Tables 11 and 12 for each combination of material properties specified in Table 9.

In each case, the initiation time to $0.5 \mathrm{~mm}$ crack extension, the time to reach a specified amount of crack growth assuming steady state conditions and the combined time involving initiation and steady state growth are given. Analysis $\mathrm{C} 1$ represents mean properties throughout, $\mathrm{C} 2$ upper bound creep strain rate and CCG behaviour and lower bound $\mathrm{CCI}, \mathrm{C} 3$ upper bound creep strain rate properties and mean CCG and CCI behaviour, $\mathrm{C} 4$ and $\mathrm{C} 5$ mean creep strain rate properties with either upper or lower bound CCG and CCI characteristics and C6 examines the influence of change in creep stress index. In all cases the creep strain rate properties determine the magnitude of $C^{*}$ and because either mean or upper bound values were chosen for these, all the initiation times are less than the average given by $\mathrm{C} 1$. Similar observations can also be made for the time to reach a specified amount of crack growth assuming steady state cracking or a combined initiation time plus steady state growth.

It is apparent that the biggest range in predicted times occurs for the C-Mn steel. This is because of the larger scatter in its uniaxial creep properties (see Fig. 4 and Table 3). It is also evident for some combinations of CCG and CCI behaviour, as for example in P91, that assumption of a combined incubation period followed by steady state growth using cyclic incubation data results in shorter lives than when steady state cracking is assumed throughout. This happens when an initiation time is chosen which gives an average cracking rate for the first $0.5 \mathrm{~mm}$ of growth which is higher than the steady state growth rate. This is not physically realistic whilst creep damage is building up at a crack tip. The implication is that a bound on $t_{i}$ should be selected such that it is never so small that it gives an initial cracking rate that is greater than the steady state value.

In order to make comparisons between deterministic and probabilistic assessments a Monte Carlo simulation has been carried out for the pipe examples using the distributions obtained from the statistical analyses. In Monte Carlo simulation, all variability of material properties were taken into account. For each example, 10000 calculations were performed to produce sufficiently accurate probability distributions. The results are shown in Figs. 14 and 15. Superimposed on these figures are the predictions of the deterministic analysis. The comparisons have been made from combined initiation plus steady state growth. It is clear in Tables 11 and 12, when mean properties are used throughout, that an approximately $50 \%$ probability of reaching the desired crack growth is achieved as is expected. In contrast, use of the worst case properties (analysis C2) corresponds with a probability of about $1 / 300$ for the C-Mn steel and 1/500 for the P91 material (i.e. less than $1 \%$ for both materials) in reaching the specific crack extensions identified in Figs. 14 and 15. The next most pessimistic prediction is given by analysis C4 for which mean uniaxial creep properties were assumed and worst case CCG (upper bound) and CCI (lower bound) data were employed. 


\section{Conclusions}

Uniaxial creep strain rate data and creep crack initiation (CCI) and creep crack growth (CCG) rate information have been presented for a $\mathrm{C}-\mathrm{Mn}$ steel at $360^{\circ} \mathrm{C}$ and a $9 \% \mathrm{Cr}$ steel (designated P91) at $625^{\circ} \mathrm{C}$. The CCI and CCG data were collected on compact tension (CT) fracture mechanics specimens and also on pressurized cracked tubes and pipes. The cracked pipes and tubes contained either an external axial rectangular slit or an axial external semi-elliptical defect. In all cases the cracking results have been interpreted using the creep fracture mechanics parameter $C^{*}$. Scatter in all the data has been analysed statistically to obtain standard deviations (SD). Good agreement has been found between the cracking behaviour of the P91 pipes and CT specimens. This has not been found to be the case for the C-Mn steel. For this material cracking in the tubes and pipes has been seen to occur faster than in the CT specimens. This has been attributed to the significant plasticity that was noted on loading and to uncertainties in estimates of reference stress for calculating $C^{*}$ for tubes and pipes containing rectangular slits.

A sensitivity analysis for the pipes has been undertaken using deterministic methods and Monte-Carlo simulations. The \pm 2 SD limits from the statistical analyses have been used to provide bounding material properties for the deterministic calculations and the basis from which variability in the properties was chosen randomly in the Monte-Carlo simulation. Times to crack initiation and to specific amounts of crack extension have been calculated and the probabilities of reaching this crack extension in a given time have been determined. It has been found that the deterministic calculations give probabilities that range from less than $1 \%$ to approximately $50 \%$. The less than $1 \%$ is achieved when the worst case combination of $\pm 2 \mathrm{SD}$ limits on properties are assumed and the $50 \%$ when mean behaviour is assumed, as is expected.

The implications of this study are that predictions of the behaviour of cracked pipes cannot be guaranteed purely from data obtained on CT specimens alone for all materials. For the C-Mn steel at least, additional tests on cracked pipe are needed to obtain appropriate scaling factors for quantitative agreement.

\section{Acknowledgements}

The authors would like to thank their partners in the European Commission funded ' $\mathrm{CCG}$ in C-Mn at 320-400 ${ }^{\circ} \mathrm{C}$ ' 1993-1997), 'HIDA' (1996-2000), and 'LICON' (1997-2001) collaborative projects, for their assistance in the testing program and provision of data.

\section{Reference}

[1] BS7910,"Guide on methods for assessing the acceptability of flaws in fusion welded structures," London, BSI, 2000.

[2] R5-British Energy, "Defect assessment code of practice for High temperature metallic components," British Energy Generation Ltd. 2000

[3] ASME Boiler \& pressure vessel code, Section XI, 1998

[4] R6, Assessment of the integrity of structures containing defects, Revision 3, British Energy Generation, 2000. 
[5] AFCEN, "Design and construction rules for mechanical components of FBR nuclear islands," RCC-MR, Appendix A16, AFCEN, Paris., 1985

[6] Ainsworth, R.A., "Some Observations on Creep Crack Growth," Int. J. of Fracture, 1982, 20, pp. 147-159

[7] Ainsworth, R.A., "The Assessment of Defects in Structure of Strain Hardening Materials," Eng. Frac. Mech., 1984, 19, pp. 633-642

[8] ASTM E1457-01, "Standard test method for measurement of creep crack growth rates in Metals," 2002

[9] Results from the "Creep Crack Growth in Carbon-Manganese," Brite/Euram Collaborative Project, European Commission 1994-1998.

[10] Results from the HIDA, "High Temperature Defect Assessment," Brite/Euram Collaborative Project, European Commission 1996-2000.

[11] Rice, J.R., "Mathematical analysis in the mechanics of fracture," in Treatise on Fracture (ed. H. Liebowitz), vol. 2, Academic Press, New York, 1968

[12] Nikbin, K.M., Smith, D.J. and Webster G.A., "Prediction of creep crack growth from uniaxial creep data," Proc. Roy. Soc. London, A 1984; 396, pp.183-197.

[13] Webster G.A. and Ainsworth R.A. High Temperature components life assessment. Chapman \& Hall, 1993

[14] Nikbin, K.M., Smith, D.J. and Webster G.A. "An engineering approach to the prediction of creep crack growth," J. of Engineering Materials and technology 1986, 108, pp.186-191

[15] Hollstein, C.B., Webster, G.A. and Djavanroodi, F., "CCG in a 1\% CrMoV steel and a 32\% Ni 20\% Cr alloy," in EGF8 Fracture Behaviour and Design of Material and Stuructures, (ed. D. Firrano), EMAS Ltd., 1990, pp.1249-1256

[16] Tabuchi, M., Yagi, K., Kubo, K. and Tanaka, C., "Effect of geometry constraint on creep crack growth behaviour of $1 \mathrm{CrMoV}$ turbine rotor steel," in Creep and Fracture of Engineering Materials and Structures (eds B. Wilshire and R.W. Evans), Institute of Metals, London, 1990, pp.729-738

[17] Penny, R.K. and Marriott, D.L., Design for Creep, McGraw-Hill, London, 1971

[18] Sim, R.G., "Evaluation of reference parameter for structures subject to creep," $J$. Mech. Eng. Sci., 1971, 13, pp.47-50

[19] Miller, A.G., "Review of limit loads of structures containing defects," Int. J. Press. Vessels Pip., 1988, 32, pp.197-327

[20] Miller, A.G. and Ainsworth, R.A. "Consistency of numerical results for powerlaw hardening materials and the accuracy of the reference stress approximation of J," Eng. Frac. Mech., 1989, 32, pp.233-247

[21] Wasmer, K, Nikbin, K. M. and Webster, G.A, "Creep Crack Initiation and Growth in Thick Section Steel Pipes under Internal Pressure," submitted for publication

[22] Nikbin, K.M., Smith, D.J. and Webster G.A. "Influence of creep ductility and state of stress on creep crack growth," in Advances in Life Prediction Methods Eds. D.A. Woodford and J.R. Whitehead, ASME, New York, pp.249-258

[23] Nikbin, K. M., "Transition effects in creep-brittle materials', Mechanics of creep brittle materials II," (Eds. A. C. F. Cocks, A.R.S. Ponter), Elsevier applied Science, 1991, pp. 14-24

[24] Austin T.S.P and Webster G.A. "Prediction of creep crack growth incubation periods," Fat. And Frac. of Engng. Mats Struct., 1992, 15, No 11, pp.1081-1090 
[25] ASME CRTD-20-1, Risk-Based Inspection - Development of Guidelines: Volume 1, General Document., ASME, New York, 1991

[26] Maskell, R. V., Fleming, A., Crawford, P. J. and Buchanan, L. W., "Creep crack growth in internally pressurised tubes," Journal of Materials at High Temperature, 1998, 15(3/4), pp.151-158.

[27] Tan, M., Celard, N.J.C., Nikbin, K.M. and Webster, G.A., "Comparison of creep crack initiation and growth in four steels tested in HIDA," Int. J. Press. Ves. \& Piping, 2001, 78, pp.737 - 747

[28] Hamata, N. Le Mat and Shibli, I. A., "Creep Crack Growth of Seam-Welded P22 and P91 Pipes with Artificial Defects. Part I: Experimental Study and Post-Test Metallography,” Int. J. Press. Ves. \& Piping, 2001, 78 (11-12), pp.819-826.

[29] Gampe, U. and Seliger, P., "Creep Crack Growth Testing of P91 and P22 Pipes Bends,” Int. J. Press. Ves. \& Piping, 2001, 78 (11-12), 859-864. 
Table 1: Chemical composition of C-Mn and P91 steel (in weight \%).

\begin{tabular}{|l|l|l|l|l|l|l|l|l|l|l|l|c|}
\hline & C & Si & Mn & P & S & Cr & Mo & Ni & Cu & Al & N & FN $^{\dagger}$ \\
\hline C-Mn steel & 0.16 & 0.28 & 0.87 & 0.014 & 0.014 & 0.09 & 0.05 & 0.2 & 0.15 & 0.019 & 0.019 & 0.0110 \\
\hline P91 & 0.091 & 0.37 & 0.41 & 0.028 & 0.013 & 8.44 & 0.92 & 0.27 & 0.04 & 0.07 & 0.038 & - \\
\hline
\end{tabular}

$\dagger \mathrm{FN}$ is calculated "Free nitrogen" for $920^{\circ} \mathrm{C}$. This heat was categorised as high free nitrogen.

Table 2: Material properties for C-Mn and P91 tested at 360 and $625^{\circ} \mathrm{C}$, respectively.

\begin{tabular}{|c|c|c|c|}
\hline Materials & Temperature & Young's modulus & $\sigma_{y}(\mathrm{MPa})$ \\
\hline $\mathrm{C}-\mathrm{Mn}$ & $360^{\circ} \mathrm{C}$ & $190 \mathrm{GPa}$ & 240 \\
\hline $\mathrm{P} 91$ & $625^{\circ} \mathrm{C}$ & $160 \mathrm{GPa}$ & 325 \\
\hline
\end{tabular}

Table 3: Mean and standard deviation of $n, \log (C)$ and $\varepsilon_{f}(\sigma$ in $\mathrm{MPa}, \dot{\varepsilon}$ in $/ \mathrm{h})$.

\begin{tabular}{|c|c|c|c|c|c|c|c|}
\hline Materials & $\begin{array}{c}\text { Temp. } \\
\left({ }^{\circ} \mathrm{C}\right)\end{array}$ & $\begin{array}{l}\text { Mean of } \\
\log (C)\end{array}$ & $\begin{array}{l}\text { Mean of } \\
n\end{array}$ & $\begin{array}{l}\text { Mean of } \\
\varepsilon_{f}(\%)\end{array}$ & $\begin{array}{l}\mathrm{SE}^{\dagger} \text { of } \\
n\end{array}$ & $\begin{array}{l}\mathrm{SD}^{\dagger} \text { of } \\
e_{\dot{\varepsilon}}\end{array}$ & $\mathrm{SD}$ of $\varepsilon_{f}$ \\
\hline $\mathrm{C}-\mathrm{Mn}$ & 360 & -37.3 & 12.5 & 18.1 & 3.36 & 0.28 & 4.0 \\
\hline P91 & 625 & -21.86 & 8.38 & 13.9 & 0.39 & 0.05 & 6.1 \\
\hline
\end{tabular}

$\uparrow$ SE and SD represent standard error and standard deviation, respectively

Table 4: Mean and standard deviation of $\phi$ and $\log (D)$ for CT CCG rate tests $\left(C^{*}\right.$ in $\mathrm{MJ} / \mathrm{m}^{2} \mathrm{~h}, \dot{a}$ in $\left.\mathrm{mm} / \mathrm{h}\right)$.

\begin{tabular}{|c|c|c|c|c|c|}
\hline Materials & Temp. & $\log (D)$ & $\phi$ & SE for $\phi$ & SD for $e_{\dot{a}}$ \\
\hline $\mathrm{C}-\mathrm{Mn}$ & $360^{\circ} \mathrm{C}$ & 1.01 & 0.89 & 0.014 & 0.18 \\
\hline $\mathrm{P} 91$ & $625^{\circ} \mathrm{C}$ & 0.15 & 0.60 & 0.030 & 0.28 \\
\hline
\end{tabular}

Table 5: Mean and standard deviation of $\phi_{i}$ and $\log \left(D_{i}\right)$ for CT CCI tests $\left(C^{*}\right.$ in $\mathrm{MJ} / \mathrm{m}^{2} \mathrm{~h}$, $t_{i}$ in $\left.\mathrm{h}\right)$.

\begin{tabular}{|c|c|c|c|c|c|}
\hline Materials & Temperature & $\log \left(D_{i}\right)$ & $\phi_{i}$ & SE for $\phi_{i}$ & SD for $e_{t_{i}}$ \\
\hline C-Mn & $360^{\circ} \mathrm{C}$ & -0.12 & -0.69 & 0.14 & 0.41 \\
\hline P91 (static) & $625^{\circ} \mathrm{C}$ & 0.39 & -0.55 & 0.15 & 0.26 \\
\hline P91 (cyclic) & $625^{\circ} \mathrm{C}$ & 0.037 & -0.55 & - & 0.17 \\
\hline
\end{tabular}


Table 6: Geometries and pressures applied to pipe and tube.

\begin{tabular}{|c|c|c|c|c|c|c|c|}
\hline \multirow{2}{*}{ Materials } & \multirow{2}{*}{ Specimen } & \multicolumn{5}{|c|}{ Component geometry $(\mathrm{mm})$} & Pressure \\
\cline { 3 - 8 } & & $R_{e}$ & $R_{i}$ & $W$ & $a_{o}$ & $a_{0} / c_{0}$ & $(\mathrm{MPa})$ \\
\hline \multirow{3}{*}{ C-Mn } & \multirow{2}{*}{ Pipe } & 63.5 & 50.5 & 13 & 1.0 & 0.04 & $69-78$ \\
\cline { 2 - 8 } & & 57.7 & 45.5 & 12 & 1.0 & 0.04 & $69-78$ \\
\cline { 2 - 8 } & Tube & 32 & 25 & 7 & 1.0 & 0.04 & $73-85$ \\
\hline P91 & Pipe & 112.5 & 92.5 & 20 & $4-7$ & $0.38-0.4$ & $10-20$ \\
\hline
\end{tabular}

$W=$ wall thickness, $R_{e}-R_{i}$

Table 7: Mean and standard deviations of $\phi$ and $\log (D)$ together with scaling factor, $\boldsymbol{\kappa}_{s}$, for pipe and tube $\left(C^{*}\right.$ in $\mathrm{MJ} / \mathrm{m}^{2} \mathrm{~h}, \dot{a}$ in $\left.\mathrm{mm} / \mathrm{h}\right)$.

\begin{tabular}{|c|c|c|c|c|c|}
\hline Materials & Temp. & $\log (D)$ & $\phi$ & SD for $e_{\dot{a}}$ & $\kappa_{s}$ \\
\hline $\mathrm{C}-\mathrm{Mn}$ & $360^{\circ} \mathrm{C}$ & 2.19 & 0.89 & 0.14 & 15.13 \\
\hline $\mathrm{P} 91$ & $625^{\circ} \mathrm{C}$ & 0.034 & 0.60 & 0.24 & 0.77 \\
\hline
\end{tabular}

Table 8: Mean and standard deviation of $\phi_{i}$ and $\log \left(D_{i}\right)$ together with scaling factor, $\kappa_{i}$, for pipe and tube $\left(C^{*}\right.$ in $\mathrm{MJ} / \mathrm{m}^{2} \mathrm{~h}, t_{i}$ in $\left.\mathrm{h}\right)$.

\begin{tabular}{|c|c|c|c|c|c|}
\hline Materials & Temp. & $\log \left(D_{i}\right)$ & $\phi_{i}$ & SD for $e_{t_{i}}$ & $\kappa_{i}$ \\
\hline C-Mn & $360^{\circ} \mathrm{C}$ & -0.96 & -0.69 & 0.17 & 0.14 \\
\hline P91 (static) & $625^{\circ} \mathrm{C}$ & 0.083 & -0.55 & 0.15 & 0.49 \\
\hline P91 (cyclic) & $625^{\circ} \mathrm{C}$ & -0.123 & -0.55 & 0.21 & 0.69 \\
\hline
\end{tabular}


Table 9: Material properties used in the deterministic method. (a) C-Mn steel, (b) P91

(a)

\begin{tabular}{|c|c|c|c|c|c|c|}
\hline \multirow{3}{*}{$\begin{array}{l}\text { Calculations } \\
\text { Analysis runs }\end{array}$} & \multirow{2}{*}{\multicolumn{2}{|c|}{$\begin{array}{l}\text { (i) }\left(\text { calculate } C^{*}\right) \\
\text { creep strain rate } \\
(\sigma \text { in } \mathrm{MPa}, \dot{\varepsilon} \text { in } / \mathrm{h})\end{array}$}} & \multirow{2}{*}{\multicolumn{2}{|c|}{$\begin{array}{l}\text { (ii) (steady state) } \\
\mathrm{CCG}-\left(C^{*} \text { in } \mathrm{MJ} / \mathrm{m}^{2} \mathrm{~h},\right. \\
\dot{a} \mathrm{~mm} / \mathrm{h})\end{array}$}} & \multirow{2}{*}{\multicolumn{2}{|c|}{$\begin{array}{l}\text { (iii) (initiation) } \\
\mathrm{CCI},\left(C^{*} \text { in } \mathrm{MJ} / \mathrm{m}^{2} \mathrm{~h},\right. \\
\left.t_{i} \text { in } \mathrm{h}\right)\end{array}$}} \\
\hline & & & & & & \\
\hline & $C$ & $n$ & $D$ & $\phi$ & $D_{i}$ & $\phi_{i}$ \\
\hline \multirow{2}{*}{$\mathrm{C} 1$} & $4.61 \times 10^{-38}$ & 12.5 & $1.34 \times 10^{2}$ & 0.89 & $1.10 \times 10^{-1}$ & -0.69 \\
\hline & mean & mean & mean & mean & mean & mean \\
\hline \multirow{2}{*}{$\mathrm{C} 2$} & $1.68 \times 10^{-37}$ & 12.5 & $3.11 \times 10^{2}$ & 0.89 & $1.62 \times 10^{-2}$ & -0.69 \\
\hline & $+2 \mathrm{SD}$ & mean & $+2 \mathrm{SD}$ & mean & $-2 \mathrm{SD}$ & mean \\
\hline \multirow{2}{*}{$\mathrm{C} 3$} & $1.68 \times 10^{-37}$ & 12.5 & $1.34 \times 10^{2}$ & 0.89 & $1.10 \times 10^{-1}$ & -0.69 \\
\hline & $+2 \mathrm{SD}$ & mean & mean & mean & Mean & mean \\
\hline \multirow{2}{*}{$\mathrm{C} 4$} & $4.61 \times 10^{-38}$ & 12.5 & $3.11 \times 10^{2}$ & 0.89 & $1.62 \times 10^{-2}$ & -0.69 \\
\hline & mean & mean & $+2 \mathrm{SD}$ & mean & $-2 \mathrm{SD}$ & mean \\
\hline \multirow{2}{*}{$\mathrm{C} 5$} & $4.61 \times 10^{-38}$ & 12.5 & $1.01 \times 10^{2}$ & 0.86 & 1.70 & -0.42 \\
\hline & mean & mean & mean & $-2 \mathrm{SD}$ & Mean & $2 \mathrm{SD}$ \\
\hline \multirow{2}{*}{ C6 } & $1.76 \times 10^{-20}$ & 5.88 & $1.01 \times 10^{2}$ & 0.86 & 1.70 & -0.42 \\
\hline & mean & $-2 \mathrm{SD}$ & mean & $-2 \mathrm{SD}$ & Mean & $2 \mathrm{SD}$ \\
\hline
\end{tabular}

(b)

\begin{tabular}{|c|c|c|c|c|c|c|c|}
\hline \multirow{3}{*}{$\begin{array}{l}\text { Calculations } \\
\text { Analysis runs }\end{array}$} & \multirow{2}{*}{\multicolumn{2}{|c|}{$\begin{array}{l}\left.\text { (i) (calculate } C^{*}\right) \\
\text { creep strain rate } \\
(\sigma \text { in } \mathrm{MPa}, \dot{\varepsilon} \text { in } / \mathrm{h})\end{array}$}} & \multirow{2}{*}{\multicolumn{2}{|c|}{$\begin{array}{l}\text { (ii) (steady state) } \\
\mathrm{CCG},\left(C^{*} \text { in } \mathrm{MJ} / \mathrm{m}^{2} \mathrm{~h},\right. \\
\dot{a} \mathrm{~mm} / \mathrm{h})\end{array}$}} & \multicolumn{3}{|c|}{ (iii) (initiation) } \\
\hline & & & & & \multicolumn{3}{|c|}{$\mathrm{CCI},\left(C^{*}\right.$ in $\mathrm{MJ} / \mathrm{m}^{2} \mathrm{~h}, t_{i}$ in $\left.\mathrm{h}\right)$} \\
\hline & C & $n$ & $D$ & $\phi$ & $\begin{array}{c}D i \\
\text { (static) }\end{array}$ & $\begin{array}{c}D i \\
\text { (cyclic) }\end{array}$ & $\phi_{i}$ \\
\hline \multirow{2}{*}{$\mathrm{C} 1$} & $1.38 \times 10^{-22}$ & 8.38 & 1.08 & 0.60 & 1.21 & 0.75 & -0.55 \\
\hline & mean & mean & mean & mean & mean & mean & mean \\
\hline \multirow{2}{*}{$\mathrm{C} 2$} & $1.74 \times 10^{-22}$ & 8.38 & 3.85 & 0.89 & 0.37 & 0.23 & -0.55 \\
\hline & $+2 \mathrm{SD}$ & mean & $+2 \mathrm{SD}$ & mean & $-2 \mathrm{SD}$ & $-2 \mathrm{SD}$ & mean \\
\hline \multirow{2}{*}{$\mathrm{C} 3$} & $1.74 \times 10^{-22}$ & 8.38 & 1.08 & 0.89 & 1.21 & 0.75 & -0.55 \\
\hline & $+2 \mathrm{SD}$ & mean & mean & mean & mean & mean & mean \\
\hline \multirow{2}{*}{$\mathrm{C} 4$} & $1.38 \times 10^{-22}$ & 8.38 & 3.85 & 0.89 & 0.37 & 0.23 & -0.55 \\
\hline & mean & mean & $+2 \mathrm{SD}$ & mean & $-2 \mathrm{SD}$ & $-2 \mathrm{SD}$ & mean \\
\hline \multirow{2}{*}{$\mathrm{C} 5$} & $1.38 \times 10^{-22}$ & 8.38 & 0.56 & 0.86 & 43.0 & 26.7 & -0.25 \\
\hline & mean & mean & mean & $-2 \mathrm{SD}$ & mean & mean & $2 \mathrm{SD}$ \\
\hline \multirow{2}{*}{ C6 } & $5.52 \times 10^{-21}$ & 7.60 & 0.56 & 0.86 & 43.0 & 26.7 & -0.25 \\
\hline & mean & $-2 \mathrm{SD}$ & mean & $-2 \mathrm{SD}$ & mean & mean & $2 \mathrm{SD}$ \\
\hline
\end{tabular}

Table 10: Loading conditions and geometries for pipe tests used in calculations.

\begin{tabular}{|l|l|l|l|l|l|l|}
\hline \multirow{2}{*}{ Materials } & \multicolumn{5}{|l|}{ Component geometry $(\mathrm{mm})$} & Pressure \\
\cline { 2 - 6 } & $R_{o}$ & $R_{i}$ & $a_{i}$ & $a_{f}$ & $a / c$ & $(\mathrm{MPa})$ \\
\hline C-Mn & 57.7 & 45.5 & 1.0 & 4.45 & 0.04 & 69.3 \\
\hline P91 & 112.5 & 92.5 & 7.5 & 10.81 & 0.38 & 16.0 \\
\hline
\end{tabular}


Table 11: Comparison of deterministic and probabilistic results for C-Mn steel.

\begin{tabular}{|c|c|c|c|c|c|c|c|c|c|}
\hline & $\begin{array}{c}\text { Experimental } \\
\text { Result }\end{array}$ & $\mathrm{C} 1$ & $\mathrm{C} 2$ & $\mathrm{C} 3$ & $\mathrm{C} 4$ & $\mathrm{C} 5$ & $\mathrm{C} 6$ & $\begin{array}{c}50 \% \text { point } \\
\text { from } \\
\text { M.C. }\end{array}$ & $\begin{array}{c}\text { 1\% point } \\
\text { from } \\
\text { M.C. }\end{array}$ \\
\hline Incubation $^{\text {ii)}},(\mathrm{h})$ & 400 & 898 & 54.1 & 366 & 133 & 418 & 209 & 955 & 56.2 \\
\hline Steady state $^{\text {iii) }},(\mathrm{h})$ & 1400 & 1140 & 155 & 358 & 488 & 1050 & 296 & 1130 & 109 \\
\cline { 1 - 7 } & 1420 & 126 & 532 & 359 & 916 & 385 & 1560 & 170 \\
\hline Combined $^{\text {iv) }},(\mathrm{h})$ & & &
\end{tabular}

i) Monte Carlo simulation (probabilistic results) at 50\% and 1\%probability level

ii) Time for $a$ to grow from 1.0 to $1.5 \mathrm{~mm}$

iii) Time for $a$ to grow from 1.0 to $4.45 \mathrm{~mm}$ using steady state CCG.

iv) Time for $a$ to grow from 1.0 to $4.45 \mathrm{~mm}$ using the combined initiation time and steady state crack growth

Table 12: Comparison of deterministic and probabilistic results for P91.

\begin{tabular}{|c|c|c|c|c|c|c|c|c|c|}
\hline $\begin{array}{l}\text { Loading and } \\
\text { condition }\end{array}$ & $\begin{array}{c}\text { Experimental } \\
\text { Result }\end{array}$ & $\mathrm{C} 1$ & $\mathrm{C} 2$ & C3 & $\mathrm{C} 4$ & $\mathrm{C} 5$ & C6 & $\begin{array}{l}\text { 50\% point } \\
\text { from } \\
\text { M.C. }\end{array}$ & $\begin{array}{l}\text { 1\% point } \\
\text { from } \\
\text { M.C. }\end{array}$ \\
\hline $\begin{array}{c}\text { Incubation }^{11)},(\mathrm{h}) \\
\text { (cyclic) }^{(\text {cyclis }}\end{array}$ & \multirow{2}{*}{530} & 562 & 151 & 494 & 172 & 532 & 532 & 563 & 137 \\
\hline $\begin{array}{c}\text { Incubation }^{\text {ii) }},(\mathrm{h}) \\
\text { (static) }\end{array}$ & & 889 & 240 & 783 & 272 & 884 & 850 & 891 & 217 \\
\hline Steady state $^{\text {iii) }},(\mathrm{h})$ & \multirow{3}{*}{2300} & 3310 & 808 & 2880 & 929 & 3190 & 2960 & 3320 & 701 \\
\hline $\begin{array}{l}\text { Combined }^{\text {iv) }},(\mathrm{h}) \\
\text { (cyclic) }\end{array}$ & & 3180 & 790 & 2770 & 907 & 3080 & 2890 & 3310 & 1050 \\
\hline $\begin{array}{c}\text { Combined }^{\text {iv })},(\mathrm{h}) \\
\text { (static) }\end{array}$ & & 3450 & 907 & 3010 & 991 & 3370 & 3170 & 3660 & 1220 \\
\hline
\end{tabular}

i) Monte Carlo simulation (probabilistic results) at 50\% and $1 \%$ probability level

ii) Time for $a$ to grow from 7.5 to $8.0 \mathrm{~mm}$

iii) Time for $a$ to grow from 7.5 to $10.81 \mathrm{~mm}$ using steady state CCG.

iv) Time for $a$ to grow from 7.5 to $10.81 \mathrm{~mm}$ using the combined initiation time and steady state crack growth 

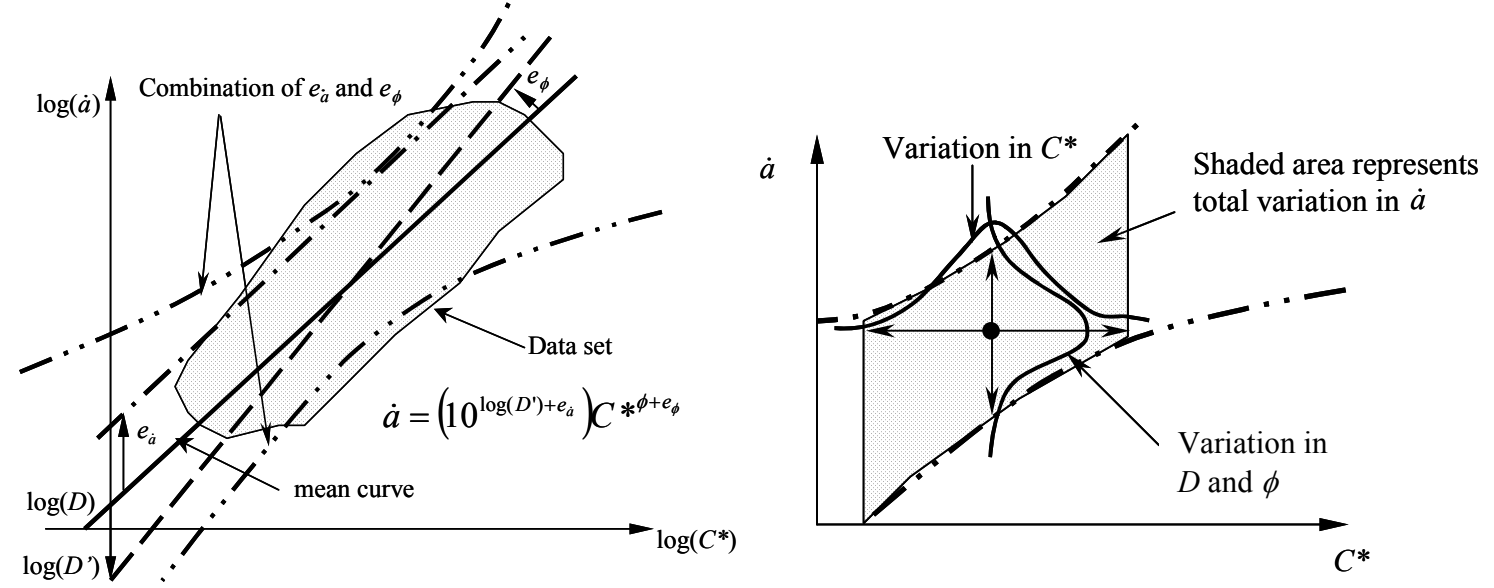

Figure 1: Error distributions from statistical Figure 2: Variation in $\dot{a}$ due to analysis of CCG rate tests. uncertainties in estimates of $D, \phi$ and $C^{*}$

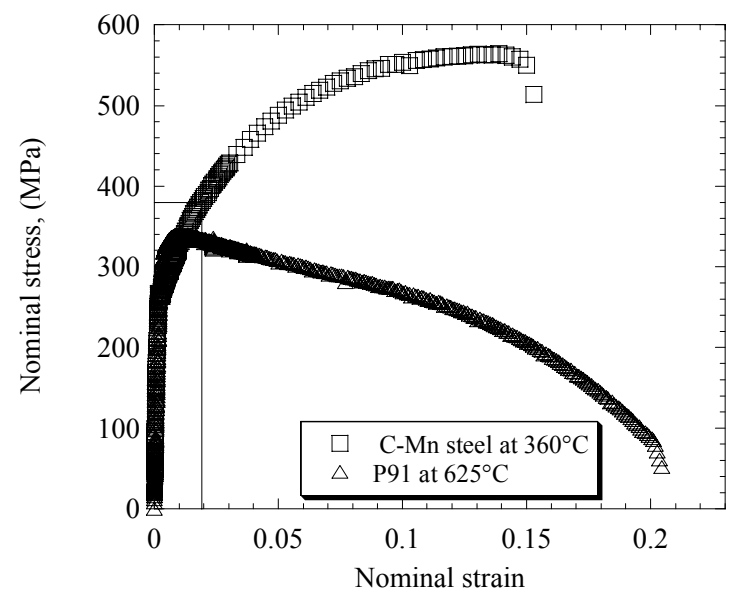

Figure 3: Stress/strain curves for C-Mn at $360^{\circ} \mathrm{C}$ and $\mathrm{P} 91$ at $625^{\circ} \mathrm{C}$.

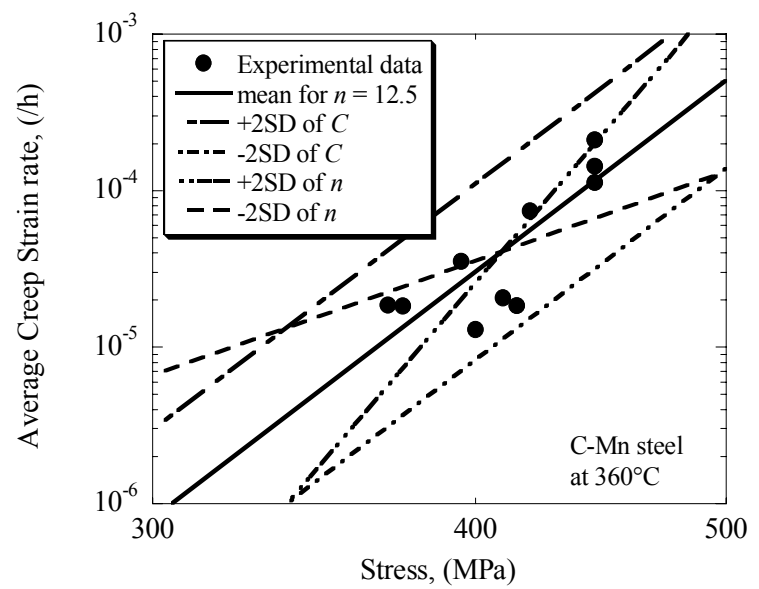

Figure 4: Average creep strain rate versus stress for C-Mn at $360^{\circ} \mathrm{C}$.

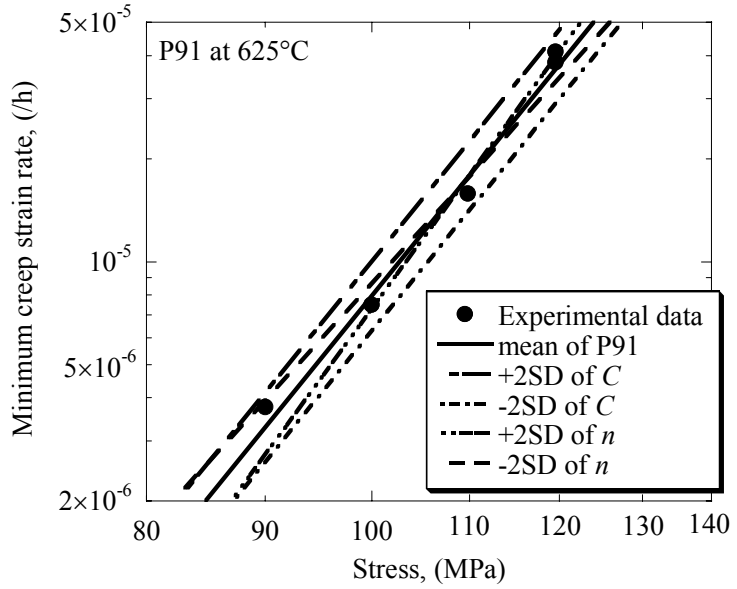

Figure 5: Minimum creep strain rate versus stress for P91 at $625^{\circ} \mathrm{C}$. 


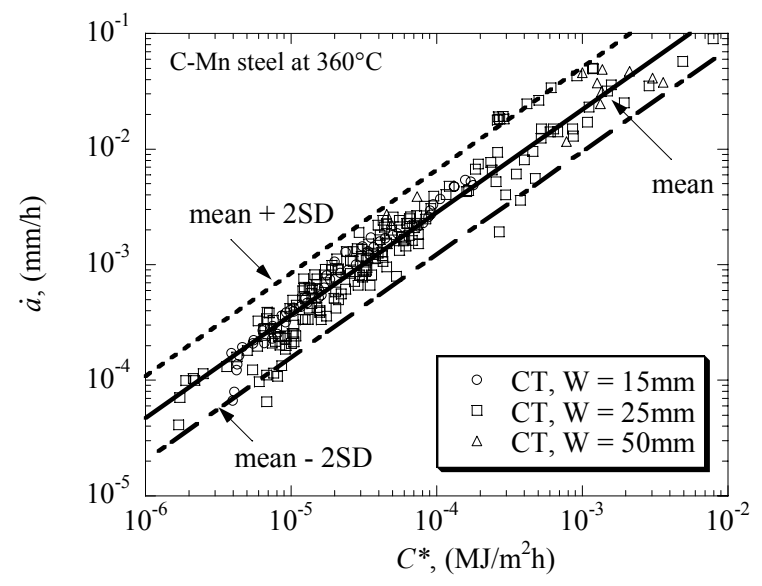

Figure 6: Steady state $\mathrm{CCG}$ rate versus $C^{*}$ for C-Mn steel CT specimen tested at $360^{\circ} \mathrm{C}$ showing the mean and $\pm 2 \mathrm{SD}$ bounds.

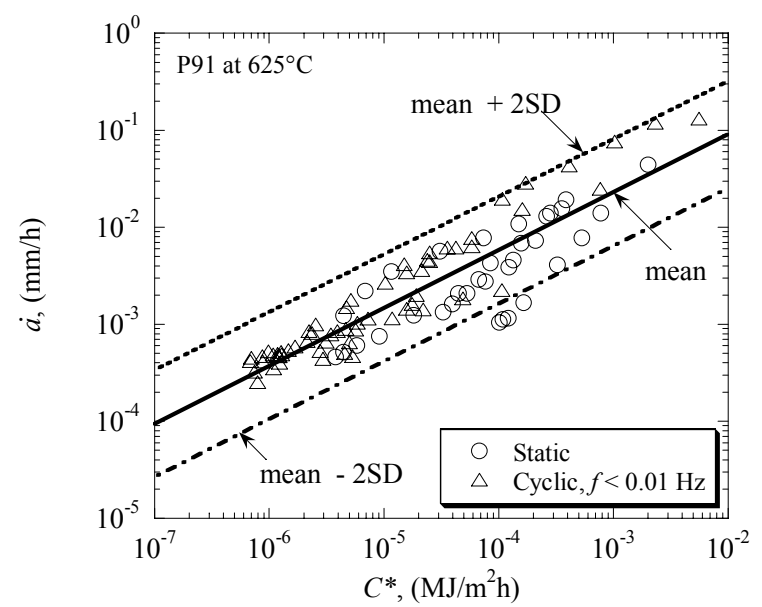

Figure 8: Steady state $\mathrm{CCG}$ rate versus $C^{*}$ for P91 CT specimens tested at $625^{\circ} \mathrm{C}$ showing the mean and \pm 2 SD bounds.

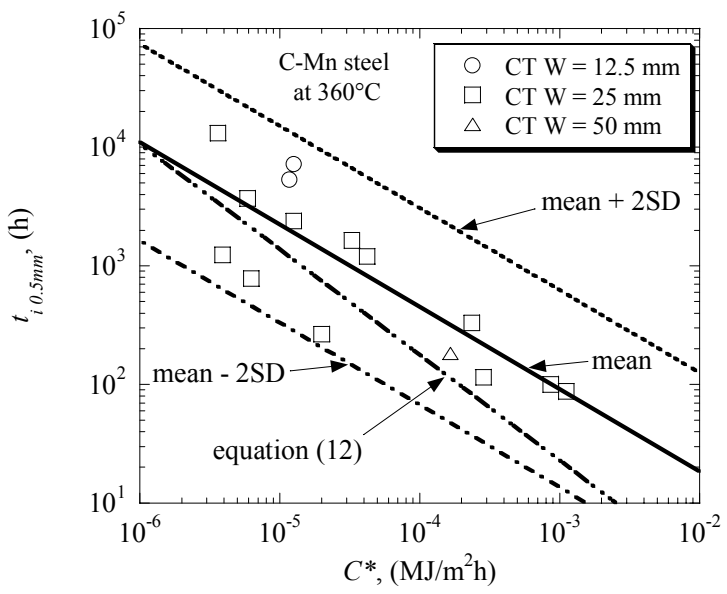

Figure 7: Initiation time for $\Delta a=0.5 \mathrm{~mm}$ versus $C^{*}$ for the $\mathrm{C}-\mathrm{Mn} \mathrm{CT}$ specimens tested at $360^{\circ} \mathrm{C}$ showing the mean and $\pm 2 \mathrm{SD}$ bounds.

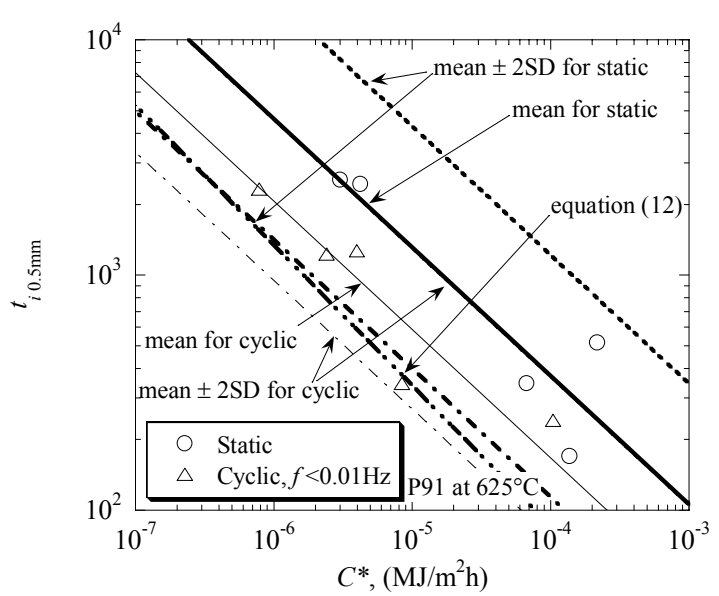

Figure 9: Initiation time to reach $\Delta a=0.5$ $\mathrm{mm}$ versus $C^{*}$ for $\mathrm{P} 91 \mathrm{CT}$ specimens tested at $625^{\circ} \mathrm{C}$ showing the mean and $\pm 2 \mathrm{SD}$ bounds. 


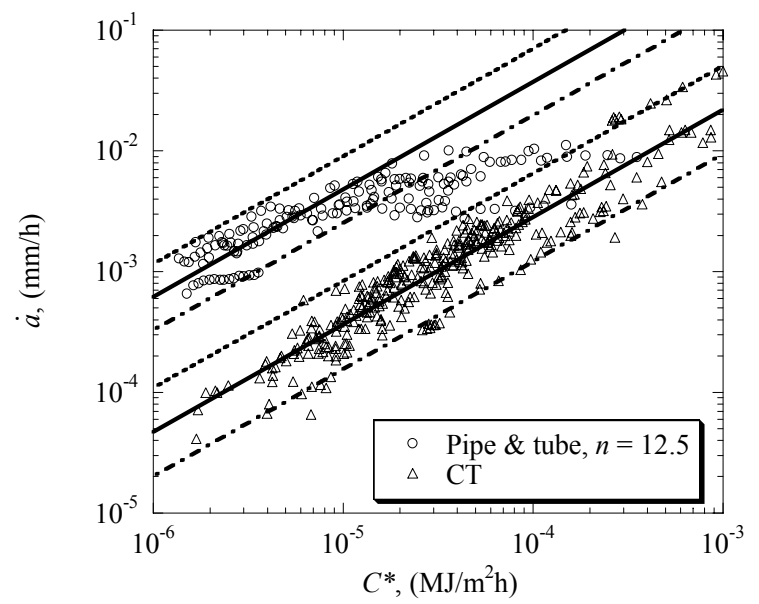

Figure 10: CCG rate versus $C^{*}$ calculated from $n=12.5$ for pipe and tube compared with $\mathrm{CT}$ specimen results.

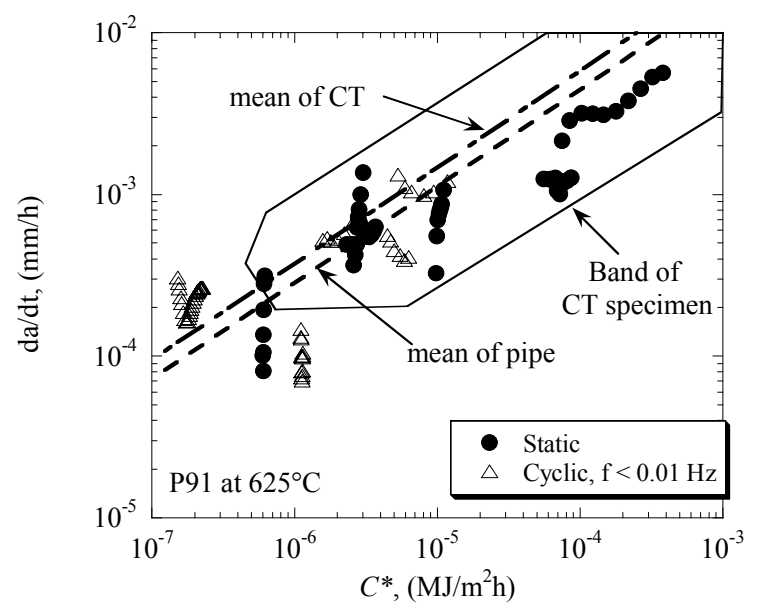

Figure 12: CCG rate for pipe compared with $\mathrm{CT}$ specimens data for $\mathrm{P} 91$ at $625^{\circ} \mathrm{C}$.

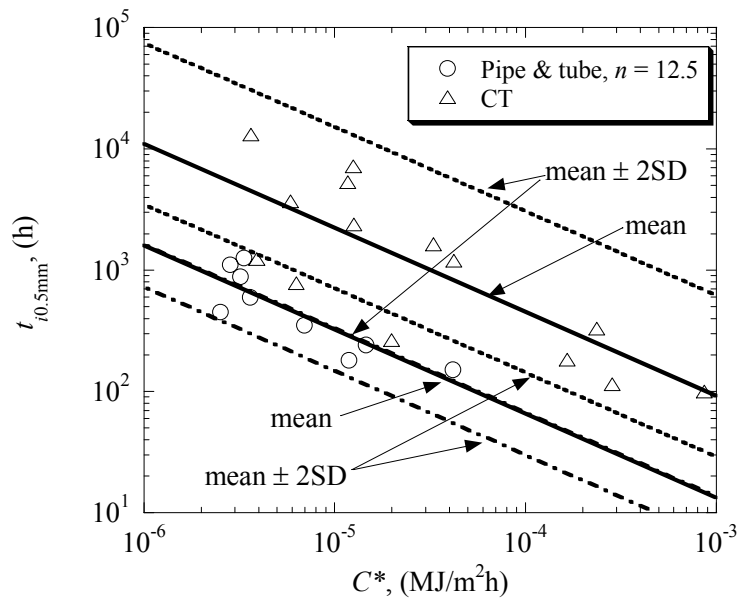

Figure 11: Initiation time against $C^{*}$ calculated from $n=12.5$ for pipe and tube compared with CT specimen results.

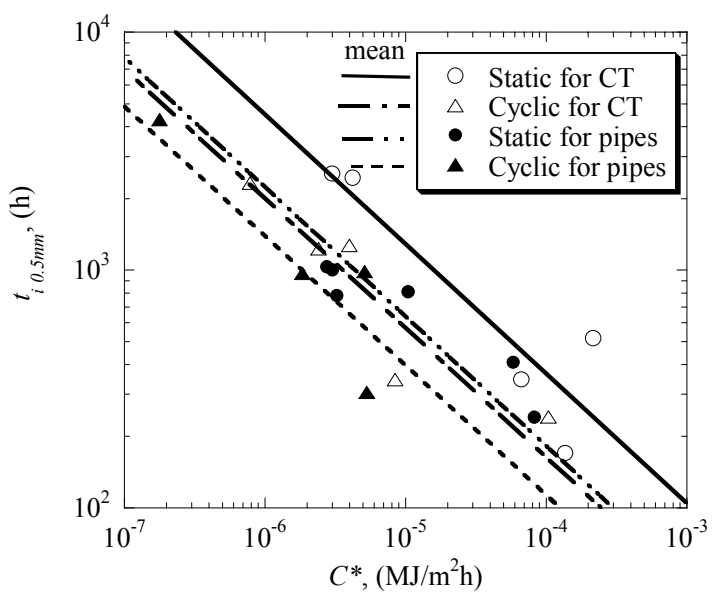

Figure 13: Initiation time for pipe with CT specimens data for $\mathrm{P} 91$ at $625^{\circ} \mathrm{C}$. 


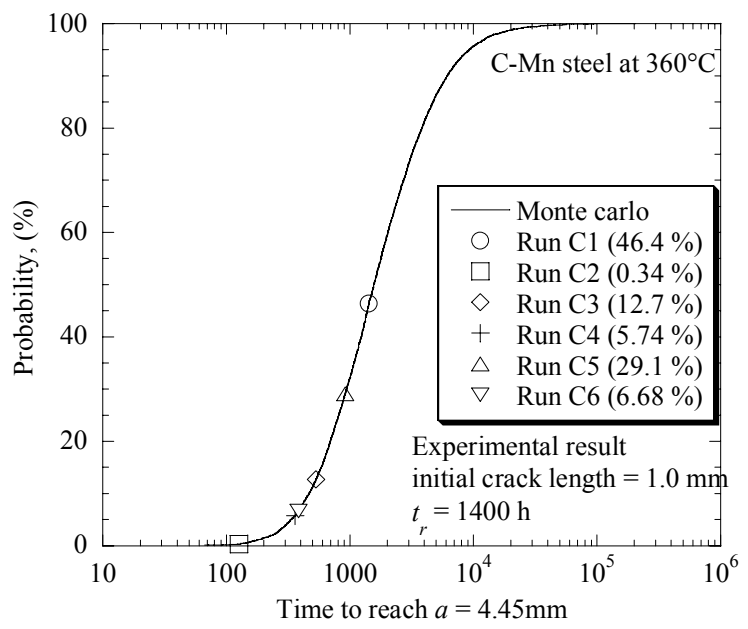

Figure 14: Comparison of deterministic and probabilistic times to reach a crack extension of $4.45 \mathrm{~mm}$ assuming combined initiation and steady state crack growth behaviour for C-Mn pipe.

(a)

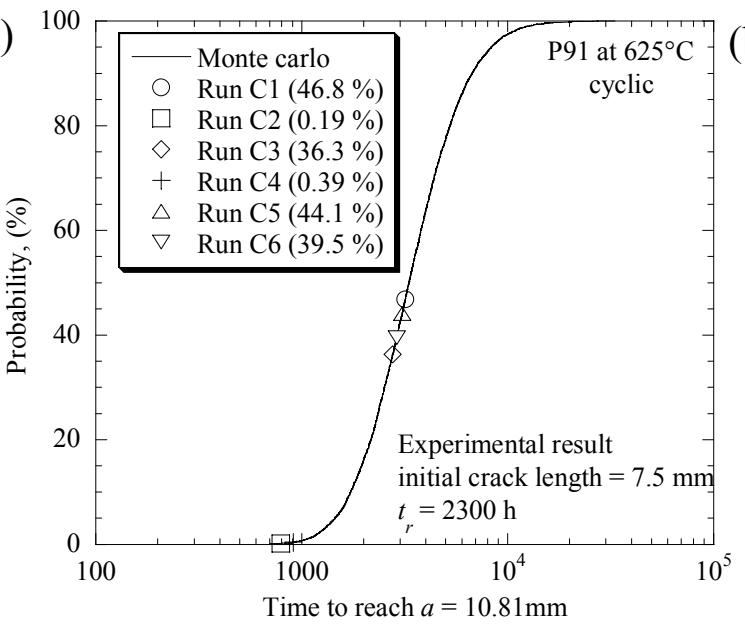

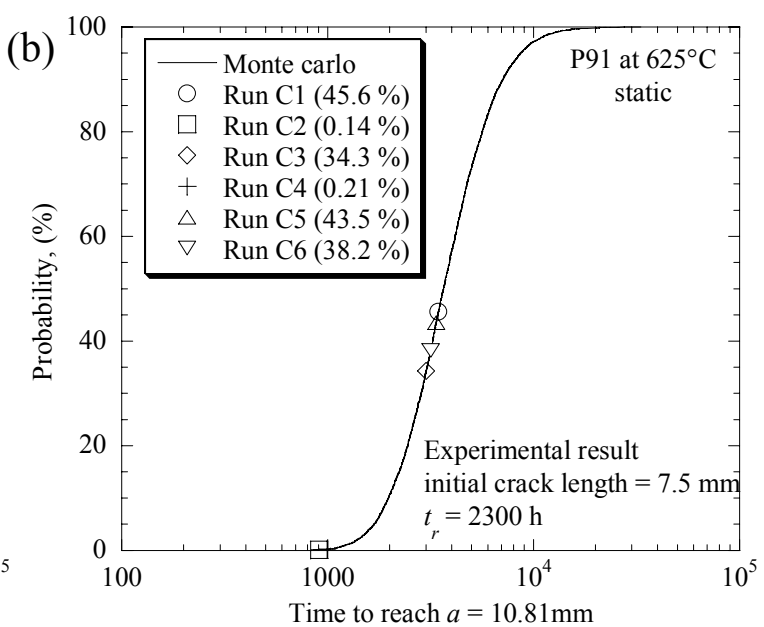

Figure 15: Comparison of deterministic and probabilistic times to reach $a=10.81 \mathrm{~mm}$ assuming a combined initiation and steady state crack growth behaviour for P91pipe. (a) cyclic and (b) static 